\title{
A Survey on Families of Binary Sequences
}

\author{
Sankhadip Roy \\ Department of Basic Science and Humanities, University of Engineering and Management, Kolkata-160, India
}

\begin{abstract}
In this correspondence, we mention several families of binary $m$-sequences which are already introduced in many articles. Most of the families have three valued non-trivial auto and cross correlations But in few cases they have five and six valued nontrivial correlations.
\end{abstract}

PACS numbers:

Keywords: Binary sequences, Quadratic Boolean functions, Correlation, Gold sequence, Gold-like sequence.

\section{INTRODUCTION}

It has been well established that families of binary sequences with low correlation have important applications in code-division multiple access (CDMA), communication systems and cryptographic system([1],[2],[3]). To check the optimality of the sequence families, we have Sidelnikov's bound([9]). It states that for any family of $k$ binary sequences of period $N_{k}$, if $k \geq N_{k}$, then

$$
R_{\max } \geq\left(2 N_{k}-2\right)^{\frac{1}{2}}
$$

where $R_{\max }$ is the maximum magnitude of correlation values except for the in-phase autocorrelation value. The well-known Gold's family ([5]) is a binary sequence family which satisfies Sidelnikov's bound. It has correlations $2^{n}-1,-1,-1 \pm 2^{\frac{n+1}{2}}$, where $n$ is odd. But Gold sequence cannot resist attacks based on Berlekamp-Massey algorithm due to its small linear span. So the Gold-like families with larger linear span were constructed.

Boztas and Kumar[4] discovered the odd case of Gold-like sequence family. The correlations of their families are identical to those of Gold sequences, namely $\left\{2^{n}-1,-1,-1 \pm 2^{\frac{n+1}{2}}\right\}$.

For even $n$, Udaya[13] introduced families of binary sequences with correlations $2^{n}-1,-1,-1 \pm 2^{\frac{n}{2}},-1 \pm 2^{\frac{n}{2}+1}$ which corresponds to even case of Gold-like sequence family.

The generalization of Gold-like sequences were done by Kim and No [7]. They have introduced GKW-like sequences by using the quadratic form technique and constructed families with correlations $2^{n}-1,-1,-1 \pm 2^{\frac{n+e}{2}}$ and $2^{n}-1,-1,-1 \pm 2^{\frac{n}{2}},-1 \pm 2^{\frac{n}{2}+e}$ respectively, where $n$ and $e$ are positive integers, $e \mid n$.

Later Wang and Qi [14] introdudced two new families $S_{1}$ and $S_{2}$ which are optimal by Sidelnikov bound.
In their work [10-12], they have combined the trace forms mentioned in $[4,7]$ and introduced new families. Some of them are actually Gold-like family.

\section{PRELIMINARIES}

Let $\mathbb{F}_{2^{n}}$ be the finite field with $2^{n}$ elements. Then the trace function from $\mathbb{F}_{2^{n}}$ to $\mathbb{F}_{2^{m}}$ is defined by

$$
\operatorname{tr}_{m}^{n}(x)=\sum_{i=0}^{\frac{n}{m}-1} x^{2^{m i}}
$$

where $x \in \mathbb{F}_{2^{n}}$ and $m \mid n$. The trace function has the following properties:

$$
\begin{aligned}
& \text { 1. } \operatorname{tr}_{m}^{n}(a x+b y)=a t r_{m}^{n}(x)+b t r_{m}^{n}(x) \text {, for all } a, b \in \\
& \quad \mathbb{F}_{2^{m}}, x, y \in \mathbb{F}_{2^{n}} ; \\
& \text { 2. } \operatorname{tr}_{m}^{n}\left(x^{2^{m}}\right)=t r_{m}^{n}(x) \text {, for all } x \in \mathbb{F}_{2^{n}} .
\end{aligned}
$$

Let $f(x)$ be a function from $\mathbb{F}_{2^{n}}$ to $\mathbb{F}_{2}$ and $\lambda \in F_{2^{n}}$. The trace transform $F(\lambda)$ of $f(x)$ is defined by

$$
F(\lambda)=\sum_{x \in \mathbb{F}_{2^{n}}}(-1)^{f(x)+t r_{1}^{n}(x \lambda)} .
$$

Definition 1. Let $x=\sum_{i=1}^{n} x_{i} \alpha_{i}$, where $x_{i} \in \mathbb{F}_{2}$ and $\alpha_{i}, i=1,2, \ldots, n$, is a basis for $\mathbb{F}_{2^{n}}$ over $\mathbb{F}_{2}$. Then the function $f(x)$ over $\mathbb{F}_{2^{n}}$ to $\mathbb{F}_{2}$ is a quadratic form if it can be expressed as

$$
f(x)=f\left(\sum_{i=1}^{n} x_{i} \alpha_{i}\right)=\sum_{i=1}^{n} \sum_{j=1}^{n} b_{i, j} x_{i} x_{j},
$$

where $b_{i, j} \in \mathbb{F}_{2^{n}}$.

The quadratic form has been well analized in [8]. We also recall that the symplectic bilinear form of a quadratic form $f(x)$ is

$$
B(x, z)=f(x)+f(z)+f(x+z) \text { for } x, z \in \mathbb{F}_{2^{n}} .
$$

Finding the dimension of the radical of a quadratic form is very crucial to find the correlations of binary sequences. 
The radical of the quadratic form $f(x)$ is the number of solutions of $x \in \mathbb{F}_{2^{n}}$ to

$$
B(x, z)=f(x)+f(z)+f(x+z)=0 \text { for all } z \in \mathbb{F}_{2^{n}} .
$$

The following lemma establishes the relation between the trace transform and the dimension of the radical of a quadratic form.

Lemma 1. ( Helleseth and Kumar [6]) Let $f(x)$ be a quadratic Boolean function on $\mathbb{F}_{2^{n}}$. If the rank of $f(x)$ is $2 h, 2 \leq 2 h \leq n$, then the distribution of the trace transform values is given by

$$
F(\lambda)= \begin{cases}2^{n-h}, & 2^{2 h-1}+2^{h-1} \text { times } \\ 0, & 2^{n}-2^{2 h} \text { times } \\ -2^{n-h}, & 2^{2 h-1}-2^{h-1} \text { times }\end{cases}
$$

where rank is the co-dimension of the radical of $f(x)$.

All the sequence families considered in this paper are constructed by using the trace function $a(x)=\operatorname{tr}_{1}^{n}(x)$ and some quadratic form $b(x)$ as follows:

$$
C=\left\{f_{i}(x) \mid 0 \leq i \leq 2^{n}, x \in \mathbb{F}_{2^{n}}^{*}\right\}
$$

where

$$
f_{i}(x)= \begin{cases}a\left(v_{i} x\right)+b(x), & 0 \leq i \leq 2^{n}-1 \\ a(x), & i=2^{n}\end{cases}
$$

and $\left\{v_{0}, v_{1}, \ldots \ldots, v_{2^{n}-1}\right\}$ is an enumeration of the elements in $\mathbb{F}_{2^{n}}$.

The correlation function between two sequences defined by $f_{i}(x)$ and $f_{j}(x)$ can be given by the function from $\mathbb{F}_{2^{n}}$ to the set of integers $\mathbb{Z}$ as

$$
R_{i, j}(\delta)=\sum_{x \in \mathbb{F}_{2^{n}}^{*}}(-1)^{f_{i}(x)+f_{j}(\delta x)}
$$

where $\delta \in \mathbb{F}_{2^{n}}^{*}=\mathbb{F}_{2^{n}} \backslash\{0\} . R_{i, j}(\delta)$ can be expressed as a trace transform

$$
\begin{aligned}
R_{i, j}(\delta) & =\sum_{x \in \mathbb{F}_{2}^{*}}(-1)^{\operatorname{tr}_{1}^{n}\left(\left[v_{i}+v_{j}\right] x\right)+g(x)} \\
& =-1+\sum_{x \in \mathbb{F}_{2^{n}}}(-1)^{\operatorname{tr}_{1}^{n}(x \lambda)+g(x)} \\
& =-1+G(\lambda)
\end{aligned}
$$

where $g(x)=b(\delta x)+b(x)$ and $\lambda=v_{i}+v_{j} \in \mathbb{F}_{2^{n}}$.

Definition 2. Let $\frac{n}{e}=m$ be odd. We define the boolean quadratic functions $p(x)$ and $q(x)$ by $p(x)=$ $\sum_{l=1}^{\frac{n}{2}-1} \operatorname{tr}_{1}^{n}\left(x^{2^{l}+1}\right), q(x)=\sum_{l=1}^{\frac{m}{2}-1} \operatorname{tr}_{1}^{n}\left(x^{2^{e l}+1}\right)$.
Lemma 2. ([4]) The associated symplectic form of $p(x)$ is

$$
B(x, z)=p(x)+p(z)+p(x+z)=\operatorname{tr}_{1}^{n}\left[z\left(\operatorname{tr}_{1}^{n}(x)+x\right)\right] .(1)
$$

Definition 3. (Boztas and Kumar [4]) For an odd integer $n=2 k+1 \geq 3$, Boztas and Kumar introduced the following family $G$ of Gold-like sequences

$$
g_{i}(x)= \begin{cases}\operatorname{tr}_{1}^{n}\left(v_{i} x\right)+p(x), & 0 \leq i \leq 2^{n}-1 \\ \operatorname{tr}_{1}^{n}(x), & i=2^{n}\end{cases}
$$

Theorem 1. (Boztas and Kumar [4]) For the family $G$ , the distribution of correlation values is given as follows:

$$
R_{i, j}(\delta)= \begin{cases}2^{n}-1, & 2^{n}+1 \text { times } \\ -1, & 2^{3 n-1}+2^{2 n}-2^{n}-2 \text { times } \\ -1+2^{k+1}, & 2^{2 n-2}\left(2^{2 k-1}+2^{k-1}\right) \text { times } \\ -1-2^{k+1}, & 2^{2 n-2}\left(2^{2 k-1}-2^{k-1}\right) \text { times }\end{cases}
$$

Lemma 3. ([7]) The associated symplectic form of $q(x)$ is

$$
B(x, z)=q(x)+q(z)+q(x+z)=\operatorname{tr}_{1}^{n}\left[z\left(\operatorname{tr}_{e}^{n}(x)+x\right)\right] .(2)
$$

Definition 4. ( Kim and No[r]) Let $\frac{n}{e}=m$ be an odd integer, where $m \geq 3$. Kim and No introduced the following sequences $S$ which generalized the previous family

$$
s_{i}(x)= \begin{cases}\operatorname{tr}_{1}^{n}\left(v_{i} x\right)+q(x), & 0 \leq i \leq 2^{n}-1 \\ \operatorname{tr}_{1}^{n}(x), & i=2^{n}\end{cases}
$$

Theorem 2. (Kim and No [7]) For the family $S$, the distribution of correlation values is given as follows:

$$
R_{i, j}(\delta)= \begin{cases}2^{n}-1, & 2^{n}+1 \text { times } \\ -1, & \left.\left(2^{n}-2^{n-e}\right)+1\right)\left(2^{2 n}-2\right) \text { times } \\ -1+2^{\frac{n+e}{2}}, & \left(2^{n-e-1}+2^{\frac{n-e-2}{2}}\right)\left(2^{2 n}-2\right) \text { times } \\ -1-2^{\frac{n+e}{2}}, & \left(2^{n-e-1}-2^{\frac{n-e-2}{2}}\right)\left(2^{2 n}-2\right)\end{cases}
$$

In their calculation to find the rank the symplectic forms (1) and (2) have been used respectively. We have used those two symplectic form in rather modified form to construct a family based on two quadratic forms $p(\lambda x)$ and $q(\zeta x)[11]$.

Definition 5. Let $\frac{n}{e}=m \geq 3$ be odd. We define the family $\mathcal{U}$ of binary sequences by 


$$
u_{i}(x)= \begin{cases}\operatorname{tr}_{1}^{n}\left(v_{i} x\right)+p(\lambda x)+q(\zeta x), & 0 \leq i \leq 2^{n}-1 \\ \operatorname{tr}_{1}^{n}(x), & i=2^{n}\end{cases}
$$

where $e$ is also odd, $\lambda, \zeta \in \mathbb{F}_{2^{e}}$ and $\lambda \neq 0, \lambda \neq \zeta$.

For the correlation property of the family $\mathcal{U}$, we have the following result.

Theorem 3. ([11]) The family $\mathcal{U}$ has the following properties:

1. The maximal absolute value of the nontrivial correlation of family $\mathcal{U}$ is bounded by $R_{\max } \leq 1+2^{\frac{n+1}{2}}$ and so the family is optimal with respect to Sidelnikov bound.

2. The correlation distribution is as follows:

$$
R_{i, j}(\delta)= \begin{cases}2^{n}-1, & 2^{n}+1 \text { times } \\ -1, & 2^{3 n-1}+2^{2 n}-2^{n}-2 \text { times } \\ -1+2^{\frac{n+1}{2}}, & \left(2^{2 n}-2\right)\left(2^{n-2}+2^{\frac{n-3}{2}}\right) \text { times } \\ -1-2^{\frac{n+1}{2}}, & \left(2^{2 n}-2\right)\left(2^{n-2}-2^{\frac{n-3}{2}}\right) \text { times }\end{cases}
$$

Definition 6. Let $\frac{n}{e}=m$ be even. We define the Boolean functions $p(x)$ and $q(x)$ by $p(x)=$ $\sum_{l=1}^{\frac{n}{2}-1} \operatorname{tr}_{1}^{n}\left(x^{2^{l}+1}\right), q(x)=\sum_{l=1}^{\frac{m}{2}-1} \operatorname{tr}_{1}^{n}\left(x^{2^{e l}+1}\right)$.

Definition 7. (Udaya [13]) For an even integer $n=$ $2 k \geq 4$, Udaya introduced the following family $G$

$g_{i}(x)= \begin{cases}\operatorname{tr}_{1}^{n}\left(v_{i} x\right)+p(x)+t r_{1}^{\frac{n}{2}}\left(x^{2^{\frac{n}{2}}+1}\right), & 0 \leq i \leq 2^{n}-1 \\ \operatorname{tr}_{1}^{n}(x), & i=2^{n} .\end{cases}$

Theorem 4. (Udaya [13]) For the family $G$, the distribution of correlation values is given as follows:

$R_{i, j}(\delta)= \begin{cases}2^{n}-1, & 2^{n}+1 \text { times } \\ -1, & 2^{2 n-1}\left(2^{n-1}+2^{n-2}\right)+2^{2 n}-2 \text { times } \\ -1+2^{k}, & \left(2^{2 n-1}-2\right)\left(2^{n-1}+2^{k-1}\right) \text { times } \\ -1-2^{k}, & \left(2^{2 n-1}-2\right)\left(2^{n-1}-2^{k-1}\right) \text { times } \\ -1+2^{k+1}, & 2^{2 n-1}\left(2^{n-3}+2^{k-2}\right) \text { times } \\ -1-2^{k+1}, & 2^{2 n-1}\left(2^{n-3}-2^{k-2}\right) \text { times. }\end{cases}$

Definition 8. ( Kim and No [7]) Let $\frac{n}{e}=m$ be an even integer, where $m \geq 4$. Kim and No introduced the following sequences $S$ with six-valued correlations.

$s_{i}(x)= \begin{cases}\operatorname{tr}_{1}^{n}\left(v_{i} x\right)+q(x)+t r_{1}^{\frac{n}{2}}\left(x^{2^{\frac{n}{2}}+1}\right), & 0 \leq i \leq 2^{n}-1 \\ \operatorname{tr}_{1}^{n}(x), & i=2^{n} .\end{cases}$
Theorem 5. ([7]) For the family $S$, the distribution of correlation values is given as follows:

$$
R_{i, j}(\delta)= \begin{cases}2^{n}-1, & 2^{n}+1 \text { times } \\ -1, & 2^{2 n-e}\left(2^{n}-2^{n-2 e}\right)+\left(2^{2 n}-2\right) \text { times } \\ -1+2^{\frac{n+2 e}{2}}, & 2^{2 n-e}\left(2^{n-2 e-1}+2^{\frac{n-2 e-2}{2}}\right) \text { times } \\ -1-2^{\frac{n+2 e}{2}}, & 2^{2 n-e}\left(2^{n-2 e-1}-2^{\frac{n-2 e-2}{2}}\right) \\ -1+2^{\frac{n}{2}}, & \left(2^{2 n}-2^{2 n-e}-2\right)\left(2^{n-1}+2^{\frac{n}{2}-1}\right) \text { times } \\ -1-2^{\frac{n}{2}}, & \left(2^{2 n}-2^{2 n-e}-2\right)\left(2^{n-1}-2^{\frac{n}{2}-1}\right) \text { times. }\end{cases}
$$

In this paper we introduce a new family $U$ which is a combination of $G$ and $S$.

Definition 9. Let $\frac{n}{e}=m \geq 4$ be even. We define the family $U$ of binary sequences by

$$
u_{i}(x)= \begin{cases}\operatorname{tr}_{1}^{n}\left(v_{i} x\right)+p(x)+q(x), & 0 \leq i \leq 2^{n}-1 \\ \operatorname{tr}_{1}^{n}(x), & i=2^{n}\end{cases}
$$

For the correlation property of the family $U$, we have the following result.

Theorem 6. ([12]) The distribution of correlation values of the family $U$ is given as when e is odd

\begin{tabular}{|l|l|}
\hline Correlation $\left(R_{i, j}(\delta)\right)$ & Number of times it appears \\
\hline $2^{n}-1$ & $2^{n}+1$ \\
-1 & $2^{3 n}+2^{2 n}-2^{n+1}+2^{e}+$ \\
& $2^{n+2 e-1}\left(2^{e-1}-2^{n-1}-1\right)-2$ \\
$-1+2^{n-\frac{e-1}{2}}$ & $\left(2^{e-2}+2^{\frac{e-3}{2}}\right)\left(2^{n+e}-2\right)$ \\
$-1-2^{n-\frac{e-1}{2}}$ & $\left(2^{e-2}-2^{\frac{e-3}{2}}\right)\left(2^{n+e}-2\right)$ \\
$-1+2^{n-e+1}$ & $\left(2^{2 e-3}+2^{e-2}\right)\left(2^{2 n}-2^{n+e}\right)$ \\
$-1-2^{n-e+1}$ & $\left(2^{2 e-3}-2^{e-2}\right)\left(2^{2 n}-2^{n+e}\right)$ \\
\hline
\end{tabular}

and when $e$ is even

\begin{tabular}{|l|l|}
\hline Correlation $\left(R_{i, j}(\delta)\right)$ & Number of times it appears \\
\hline $2^{n}-1$ & $2^{n}+1$ \\
-1 & $2^{3 n}+2^{2 n}-2^{n+1}+2^{e+1}+$ \\
& $2^{n+e-2}\left(3 \cdot 2^{e-1}-2^{n+2}-3\right)-2$ \\
$-1+2^{n-\frac{e}{2}}$ & $\left(2^{e-1}+2^{\frac{e-2}{2}}\right)\left(2^{n+e-1}+2^{n}-2\right)$ \\
$-1-2^{n-\frac{e}{2}}$ & $\left(2^{e-1}-2^{\frac{e-2}{2}}\right)\left(2^{n+e-1}+2^{n}-2\right)$ \\
$-1+2^{n-\frac{e-2}{2}}$ & $\left(2^{e-3}+2^{\frac{e-4}{2}}\right)\left(2^{n+e-1}-2^{n}\right)$ \\
$-1-2^{n-\frac{e-2}{2}}$ & $\left(2^{e-3}-2^{\frac{e-4}{2}}\right)\left(2^{n+e-1}-2^{n}\right)$ \\
$-1+2^{n-e}$ & $\left(2^{2 e-1}+2^{e-1}\right)\left(2^{2 n}-2^{n+e}\right)$ \\
$-1-2^{n-e}$ & $\left(2^{2 e-1}-2^{e-1}\right)\left(2^{2 n}-2^{n+e}\right)$ \\
\hline
\end{tabular}


Definition 10. Let $n$ be odd, $\delta_{1} \in \mathbb{F}_{2^{n}} \backslash\{0,1\}$. We define $p(x)=\sum_{l=1}^{\frac{n-1}{2}} \operatorname{tr}_{1}^{n}\left(x^{2^{l}+1}+\left(\delta_{1} x\right)^{2^{l}+1}\right)$, $q(x)=p(x)+p\left(\delta_{2} x\right)$, where $\delta_{2} \in \mathbb{F}_{2^{n}} \backslash\{0,1\}$ and $\delta_{1} \neq \delta_{2}$.

Using the rank of $p(x)$ and $q(x)$, Wang and Qi [14] has introduced the following result.

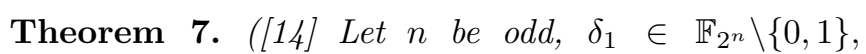
$p(x)=\sum_{l=1}^{\frac{n-1}{2}} \operatorname{tr}_{1}^{n}\left(x^{2^{l}+1}+\left(\delta_{1} x\right)^{2^{l}+1}\right)$. Then the family $S_{1}=\left\{s_{1, j} \mid j=0,1, \ldots, 2^{n}\right\}$ given by

$$
s_{1, j}(x)= \begin{cases}\operatorname{tr}_{1}^{n}\left(v_{j} x\right)+p(x), & 0 \leq i \leq 2^{n}-1 \\ \operatorname{tr}_{1}^{n}(x), & i=2^{n} .\end{cases}
$$

has the following correlation distribution:

\begin{tabular}{|l|l|}
\hline Correlation $\left(R_{i, j}(\delta)\right)$ & Number of times it appears \\
\hline $2^{n}-1$ & $2^{n}+1$ \\
-1 & $2^{3 n-1}+2^{3 n-4}-2^{3 n-6}+2^{2 n}-2^{n}-2$ \\
$-1+2^{\frac{n+1}{2}}$ & $\left(2^{n-2}+2^{\frac{n-3}{2}}\right)\left(2^{2 n}-2^{2 n-3}-2\right)$ \\
$-1-2^{\frac{n+1}{2}}$ & $\left(2^{n-2}-2^{\frac{n-3}{2}}\right)\left(2^{2 n}-2^{2 n-3}-2\right)$ \\
$-1+2^{\frac{n+3}{2}}$ & $\left(2^{n-4}+2^{\frac{n-5}{2}}\right) 2^{2 n-3}$ \\
$-1-2^{\frac{n+3}{2}}$ & $\left(2^{n-4}-2^{\frac{n-5}{2}}\right) 2^{2 n-3}$ \\
\hline
\end{tabular}

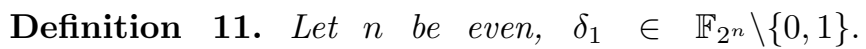
We define $p_{1}(x)=\operatorname{tr}_{1}^{n}\left(x^{2^{n / 2}+1}+\left(\delta_{1} x\right)^{2^{n / 2}+1}\right)+$ $\sum_{l=1}^{\frac{n}{2}-1} \operatorname{tr}_{1}^{n}\left(x^{2^{l}+1}+\left(\delta_{1} x\right)^{2^{l}+1}\right)$,

$q_{1}(x)=p_{1}(x)+p\left(\delta_{2} x\right)$, where $\delta_{2} \in \mathbb{F}_{2^{n}} \backslash\{0,1\}$ and $\delta_{1} \neq \delta_{2}$.

Using the rank of $p_{1}(x)$ and $q_{1}(x)$, Wang and Qi [14] has introduced another new family.

Theorem 8. Let $n$ be even, $\delta_{1} \in \mathbb{F}_{2^{n}} \backslash\{0,1\}, q(x)=$ $\operatorname{tr}_{1}^{n}\left(x^{2^{n / 2}+1}+\left(\delta_{1} x\right)^{2^{n / 2}+1}\right)+\sum_{l=1}^{\frac{n}{2}-1} t r_{1}^{n}\left(x^{2^{l}+1}+\left(\delta_{1} x\right)^{2^{l}+1}\right)$, Then the family $S_{2}=\left\{s_{2, j} \mid j=0,1, \ldots, 2^{n}\right\}$ given by

$$
s_{2, j}(x)= \begin{cases}\operatorname{tr}_{1}^{n}\left(v_{j} x\right)+p(x), & 0 \leq i \leq 2^{n}-1 \\ \operatorname{tr}_{1}^{n}(x), & i=2^{n} .\end{cases}
$$

has the following correlation distribution:

\begin{tabular}{|l|l|}
\hline Correlation $\left(R_{i, j}(\delta)\right)$ & Number of times it appears \\
\hline $2^{n}-1$ & $2^{n}+1$ \\
-1 & $2^{3 n-1}-2^{3 n-3}+2^{3 n-6}-2^{3 n-8}+$ \\
& $2^{2 n}-2$ \\
$-1+2^{\frac{n}{2}}$ & $\left(2^{n-1}+2^{\frac{n}{2}-1}\right)\left(2^{2 n-1}-2\right)$ \\
$-1-2^{\frac{n}{2}}$ & $\left(2^{n-1}-2^{\frac{n}{2}-1}\right)\left(2^{2 n-1}-2\right)$ \\
$-1+2^{\frac{n}{2}+1}$ & $\left(2^{n-3}+2^{\frac{n}{2}-2}\right)\left(2^{2 n-1}-2^{2 n-4}\right)$ \\
$-1-2^{\frac{n}{2}+1}$ & $\left(2^{n-3}-2^{\frac{n}{2}-2}\right)\left(2^{2 n-1}-2^{2 n-4}\right)$ \\
$-1+2^{\frac{n}{2}+2}$ & $\left(2^{n-5}+2^{\frac{n}{2}-3}\right) 2^{2 n-4}$ \\
$-1-2^{\frac{n}{2}+2}$ & $\left(2^{n-5}-2^{\frac{n}{2}-3}\right) 2^{2 n-4}$ \\
if $\operatorname{tr}_{1}^{n}\left(\left(1+\delta_{1}\right)^{-1}\right)=0$ and
\end{tabular}

\begin{tabular}{|l|l|}
\hline Correlation $\left(R_{i, j}(\delta)\right)$ & Number of times it appears \\
\hline $2^{n}-1$ & $2^{n}+1$ \\
-1 & $2^{3 n-1}-2^{3 n-3}+2^{3 n-6}-2^{3 n-8}+$ \\
& $2^{2 n}-2$ \\
$-1+2^{\frac{n}{2}}$ & $\left(2^{n-1}+2^{\frac{n}{2}-1}\right) 2^{2 n-1}$ \\
$-1-2^{\frac{n}{2}}$ & $\left(2^{n-1}-2^{\frac{n}{2}-1}\right) 2^{2 n-1}$ \\
$-1+2^{\frac{n}{2}+1}$ & $\left(2^{n-3}+2^{\frac{n}{2}-2}\right)\left(2^{2 n-1}-2\right)$ \\
$-1-2^{\frac{n}{2}+1}$ & $\left(2^{n-3}-2^{\frac{n}{2}-2}\right)\left(2^{2 n-1}-2\right)$ \\
\hline
\end{tabular}

if $\operatorname{tr}_{1}^{n}\left(\left(1+\delta_{1}\right)^{-1}\right)=1$

\section{CONCLUSION}

In this article we have seen a quick survey on families of binary $m$-sequences with 3,4 and more non-trivial correlation values. More results can be found in recent literature. But in order to achieve bigger linear span many families of quartenary sequences have been introduced.

\section{ACKNOWLEDGEMENT}

I would like to thank my colleagues from department of Basic Science and Humanities, University of Engineering and Management, Kolkata for their valuable advice leading to writing this paper.
[1] Ziemer, R.,Peterson, R., Digital Communication and spectrum communication systems., McMilian, New York (1985).

[2] Gong, G., New designs for signal sets with low cross correlation, balance property, and large linear span: $G F(p)$ case, IEEE Trans. Inform. Theory, 48 (2002),2847-2867.

[3] Fan, P.Z., Darnell, M., Sequence Design for Communications Applications, John Wiley, Chichester (1996)

[4] Boztas, S., Kumar, P.V., Binary sequences with Gold-like correlation but larger linear span, IEEE Trans. Inform. 
Theory, 40 (1994),532-537.

[5] Gold, R., Maximal recursive sequences with 3-valued recursive cross-correlation functions, IEEE Trans. Inform. Theory, 14 (1968), 154-156.

[6] Helleseth, T. and Kumar, P.V., "SSequences with low correlation,"in Handbook of Coding Theory, V.S. Pless and W.C. Huffman, Eds. Amsterdam, The Netherlands: Elsevier (1998).

[7] Kim, S.H., and No, J.S., New families of Binary Sequences with low correlation, IEEE Trans. Inform. Theory, 49,No.11 (2003), 3059-3065.

[8] Lidl, R. and Niederreiter ,W., Finite Fields, Encyclopedia of Mathematics and its Application, Vol 20, Cambridge University Press , Cambridge , 1997.

[9] Sidelnikov, V.M. On mutual correlations of sequences, Soviet Math. Dokl. 12, 197-201 (1971).

[10] Tang, X., Helleseth,T., Hu,L., Jiang,W., A new family of Gold-like sequences, S.W.Golomb et al.(Eds.):SSC
2007.LNCS 4893,(2007), 62-69.

[11] Roy, S. Another New Family of Gold-Like Sequences,American Journal of Physical Sciences and Applications, 1(1),24-28(2020)

[12] Roy, S. Another New Family of Binary Sequences with Six or eight-valued Correlations, American Journal of Applied Mathematics and Computing, Vol 1, Issue 2,19-23, 2020

[13] Udaya, P., "Polyphase and frequency hopping sequences obtained from finite rings", Ph.D. dissertation, Dept. Elec. Eng.,Indian Inst. Technol., Kanpur, 1992.

[14] Wang JS., Qi WF., Four Families of Binary Sequences with Low Correlation and Large Linear Complexity. In: Pei D., Yung M., Lin D., Wu C. (eds) Information Security and Cryptology. Inscrypt 2007. Lecture Notes in Computer Science, vol 4990. Springer, Berlin, Heidelberg. 